\title{
Study on Operation Control Strategy of Adjacent Pump Stations in Long Distance Multistage Pumping Station Control System
}

\author{
Xu Xuejun ${ }^{1, a}$, Yan Ying ${ }^{2, b}$, Ma qingqing ${ }^{3, c}$ \\ ${ }^{1}$ School of Hydropower and Information Engineering,Huazhong University of Science and \\ Technology, Wuhan, Hubei 430074, P. R. China \\ ${ }^{2}$ Patent Examination Cooperation Hubei Center of the Patent Office, SIPO, \\ Wuhan,Hubei 430205, China \\ ${ }^{3}$ School of Hydropower and Information Engineering, Huazhong University of Science and \\ Technology, Wuhan, Hubei 430074, P. R. China
}

cbbw@hust.edu.cn

Keywords: Long-distance water. time difference control. power-on program.

Abstract. Aiming at the operation control of adjacent pump stations in the multi-stage pumping station of a small buffer pool, a transient mathematical model of pipeline flow was established by hydraulic calculation in the pipeline to calculate the start-up time difference between pumping stations at different levels. AFT impulse software was used to measure the time difference The validity of the control mode is verified. Based on this, the time difference between start-up allowable time of multi-stage pumping station with different buffer pools is calculated and analyzed, which provides a theoretical basis and data support for the joint control of long-distance multi-stage pumping stations with small buffer.

\section{Research background}

The operation control of long-distance multi-stage pumping station in our country is divided into two levels, which are adjacent pumping station and single pumping station respectively. At present, domestic and foreign experts and scholars have made a series of optimization control in adjacent pumping station : In 2003, according to the characteristics of the hydraulic transition process of Dongshen Water Supply Project, Pan Zhiquan [1] established a mathematical model for the calculation of the joint hydraulic transition process of pipe, canal and pump system. In 2007, Han Yancheng [2,3] Shandong Province Jiaodong water transfer project, the application of visual computing program to simulate long-distance complex cascade water supply hydraulic system operation, long-distance multi-stage pumping station and gravity flow channel operation mode was discussed; Tianjin University in 2016 Gao Xueping[4] established the multi-dimensional mathematical model of the inter-stage section of the cascade pump station to simulate the water level changes under different water diversion conditions and determined the time difference between the opening of the adjacent and adjacent cascade pump stations. Based on the two variables of annual water diversion and initial water diversion, the quantitative relationship between critical time difference and critical water level is proposed by Levenberg-Marquardt algorithm and Universal Global Optimization algorithm This is the judgment condition for the opening of the cascade pump station, and the inter-adjacent pumping station is mainly the problem of inter-level cooperation and joint scheduling of the multi-stage pumping station project. In the face of dynamically changing flow and lift requirements, Open and stop to meet the relevant mission requirements, frequently open and stop the pump equipment costs will increase the cost of the same time, the operation of the pump station neglects the time delay of the water subsystem will also bring a certain loss of power costs,

(Note: This paper has been supported by the Innovative Rearch Fund of HUST)

This paper aims to control the operation of adjacent pumping stations, and formulate the corresponding joint scheduling and control scheme to achieve the goal of efficient and economical operation of the system. 


\section{Pumping station control time analysis}

Outflow at the upper level Q1 out = Inflow of the next pumping station into the pool $\mathrm{Q}_{2 \text { in }}+$ Interstage flow loss $\mathrm{Q}$ loss

Due to the long distance between the two stations and the small buffer pool volume, the interaction between the pumping stations and the lag of their flow influence the safe operation of the system.

In the actual operation control process, in accordance with the current general control strategy, the pump stations at all levels are generally opened at the same time. When the flow rate is specified, the longer the distance between two pumping stations $\mathrm{A}$ and $\mathrm{B}$, the larger the required buffer pool volume is Will result in buffer pool required area is also larger. In this scenario, it is highly probable that the incoming water will flow upstream and the water level in the buffer pool of the station has dropped below the minimum operating water level, causing the pump to be forced to stop, reducing the service life of the equipment and causing cavitation in the pump Vibration, causing the water pump suction pipe off, endangering the safe operation of the pump station.

Therefore, it is not enough to consider only the propagation time when a water surge front of a higher-level pump station arrives at a lower-level pump station. It should be based on the fact that the opening time does not cause the buffer tank water level to be lower than the actual control water level, The water storage capacity to meet the appropriate design flow as a sufficient condition to the water level at the pump station striker reach the water level of the next pump station began to rise as the necessary conditions to calculate the water pump station to advance to the lower level pump station time as Adjacent pumping station parameters to open the time difference to ensure long-distance small buffer pool water project safe operation.

\section{Adjacent pumping stations between the water system modeling}

The buffer tank of the adjacent pumping station is all through the pressure pipe to complete the water, so at this time the long-distance multi-stage pumping station interstage pipelines hydraulics model can refer to no pressure chamber hydraulic system has pressure Water diversion pipeline model, as shown below, $\mathrm{H}$ for the level of net pumping head lift.

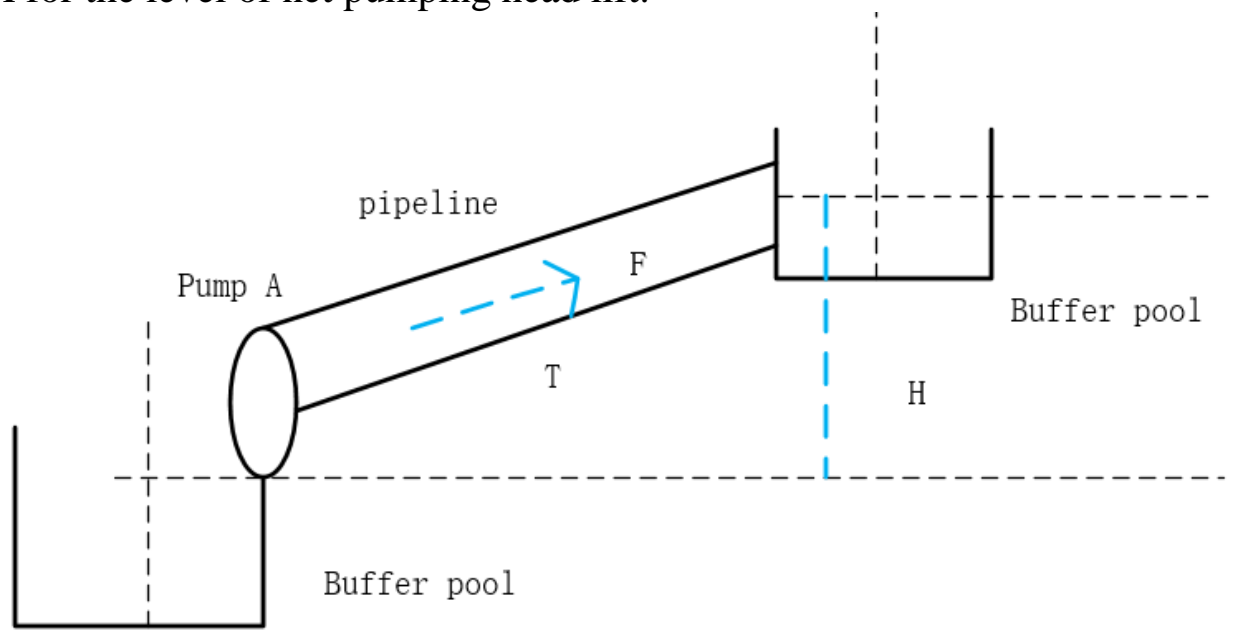

Fig. 1

the pipeline surge propagation time

$$
T_{p}=\frac{L}{b}
$$


$\mathrm{T}_{\mathrm{P}}$ :Pressure pipeline surge propagation time; L: Pressure pipe length; b: Pipeline water wave propagation speed

the water hammer wave speed inside the pipe.The water hammer wave velocity is analyzed by a one-dimensional model in the pipeline:

$$
T_{P}=\frac{L}{b}=L * \sqrt{\frac{\rho}{k}\left(1+\frac{\varphi * k}{f * E}\right)}
$$

The reason that the adjacent two pumping stations should have a reasonable time difference of pump-on should mean that the water pumping capacity of the sub-pumping station within this interval is less than or equal to the storage capacity of the buffer tank, that is, the volume of water body above the minimum water level of the buffer tank design. Which can be derived from the adjacent upper and lower pumping station opening time difference $t$ formula.

According to the adjacent level pumping station water balance principle can be obtained:

$$
\begin{gathered}
V=Q_{1} t+\left(Q_{1}-Q_{\varepsilon}\right) T_{p} \\
t=\left[V-\left(Q_{1}-Q_{\varepsilon}\right) T_{p}\right] / Q_{1}
\end{gathered}
$$

$\mathrm{V}$ : The maximum water storage volume above the lowest water level in the buffer pool between two adjacent pumping stations; Tp: Superior pump station wave forward spread to the sub-pumping station time.

\section{Analysis and Verification of Water Conveyance Process of Adjacent Pumping Station}

According to an example of a water conveyance project, if the pipe is a steel pipe, its elastic modulus is $206 \mathrm{GPa}$.

Assuming the first two pump stations between the pipe diameter of $1.1 \mathrm{~m}$, pipe length of $11800 \mathrm{~m}$, wall thickness of $0.01 \mathrm{~m}$;

The pipe diameter of the water conveyance pipeline between the second and third class pumping stations is $1 \mathrm{~m}$, the pipe length is $4450 \mathrm{~m}$, the pipe wall thickness is $0.01 \mathrm{~m}$;

The pipe diameter of the water conveyance pipeline between the 34 pumping stations is $0.5 \mathrm{~m}$, the pipe length is $2200 \mathrm{~m}$ and the pipe wall thickness is $0.015 \mathrm{~m}$.

The size of the buffer pool between the first and second pumping stations is $24 \mathrm{~m} * 5 \mathrm{~m}$ (length $*$ width); the buffer pool size of the second and third pumping stations is $14 \mathrm{~m} * 5 \mathrm{~m}$;

Three or four pumping stations between the buffer pool size of $11.4 \mathrm{~m} * 11.4 \mathrm{~m}$.

According to the above formula 2, the propagation time of the surge in the pipe of the adjacent pumping station corresponding to each pipe section at this moment is as follows:

\begin{tabular}{|c|c|}
\hline Pipe section & Surge propagation time (s) \\
\hline $\begin{array}{c}\text { Between the first and second } \\
\text { pumping stations }\end{array}$ & 28.415 \\
\hline $\begin{array}{c}\text { Between the second and thrid } \\
\text { pumping stations }\end{array}$ & 10.716 \\
\hline $\begin{array}{c}\text { Between the third and fourth pumping } \\
\text { stations }\end{array}$ & 5.298 \\
\hline
\end{tabular}

By comparing and analyzing the water hammer changes in two adjacent pumping stations when the adjacent two pumping stations are turned on simultaneously and the time difference calculated in this paper is turned on (adjusted) successively The proposed method of controlling the time difference is effective. AFT (Applied Flow Technology) impulse was chosen to numerically simulate the hydraulic 
transients of the pipelines connected to the pumping station during service changes. It is a powerful water hammer modeling software tool that can be used to simulate various Transient Flow Behavior of Piping System [5,6].

AFT impulse analysis of the above conditions at the same time increase the flow and increase the flow rate after a certain time difference, the pressure inside the pipeline between the pumping station, where the maximum pressure in the three sections of the pipeline as the representative of the maximum pressure for analysis, the results are as follows Figure 2, Figure 3 shows.

Fig.2 Adjacent pumping stations increase the flow rate at the same time

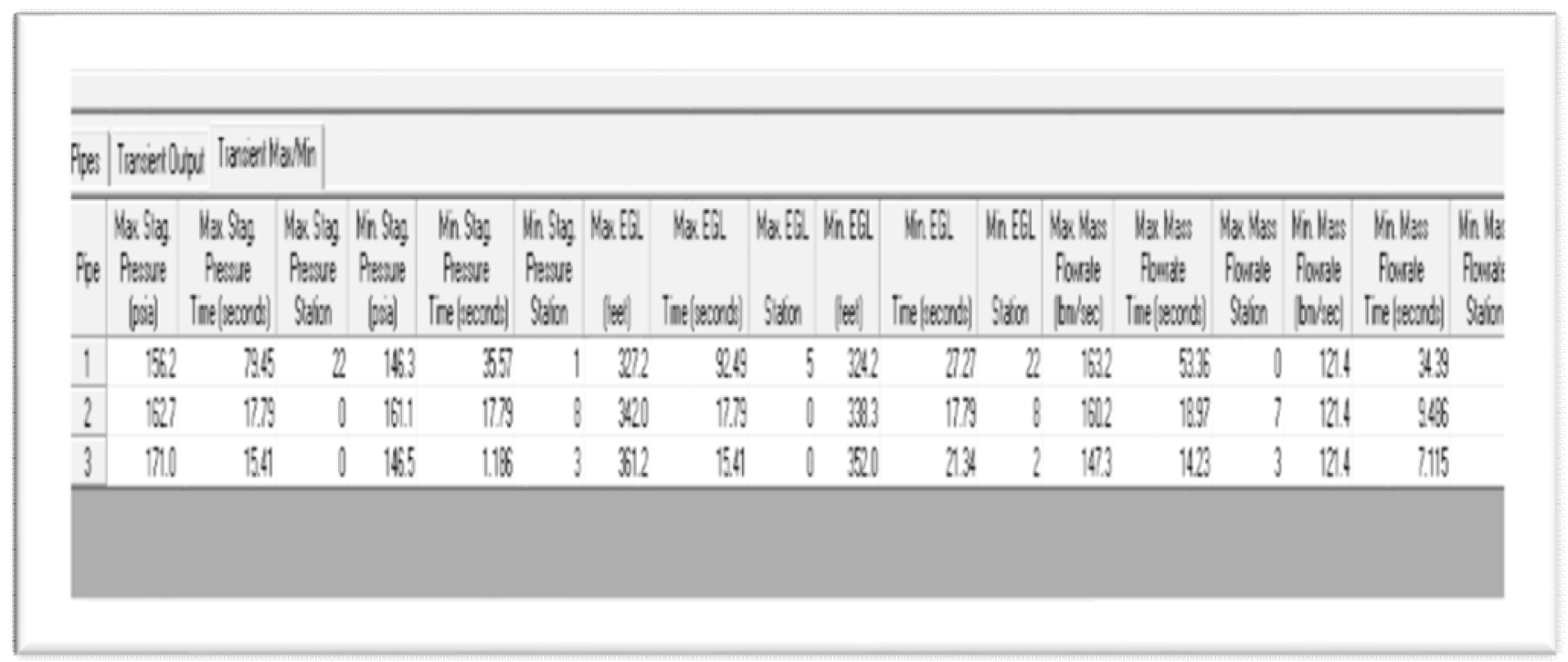

Fig.3 Adjacent pumping stations to increase the flow of time interval

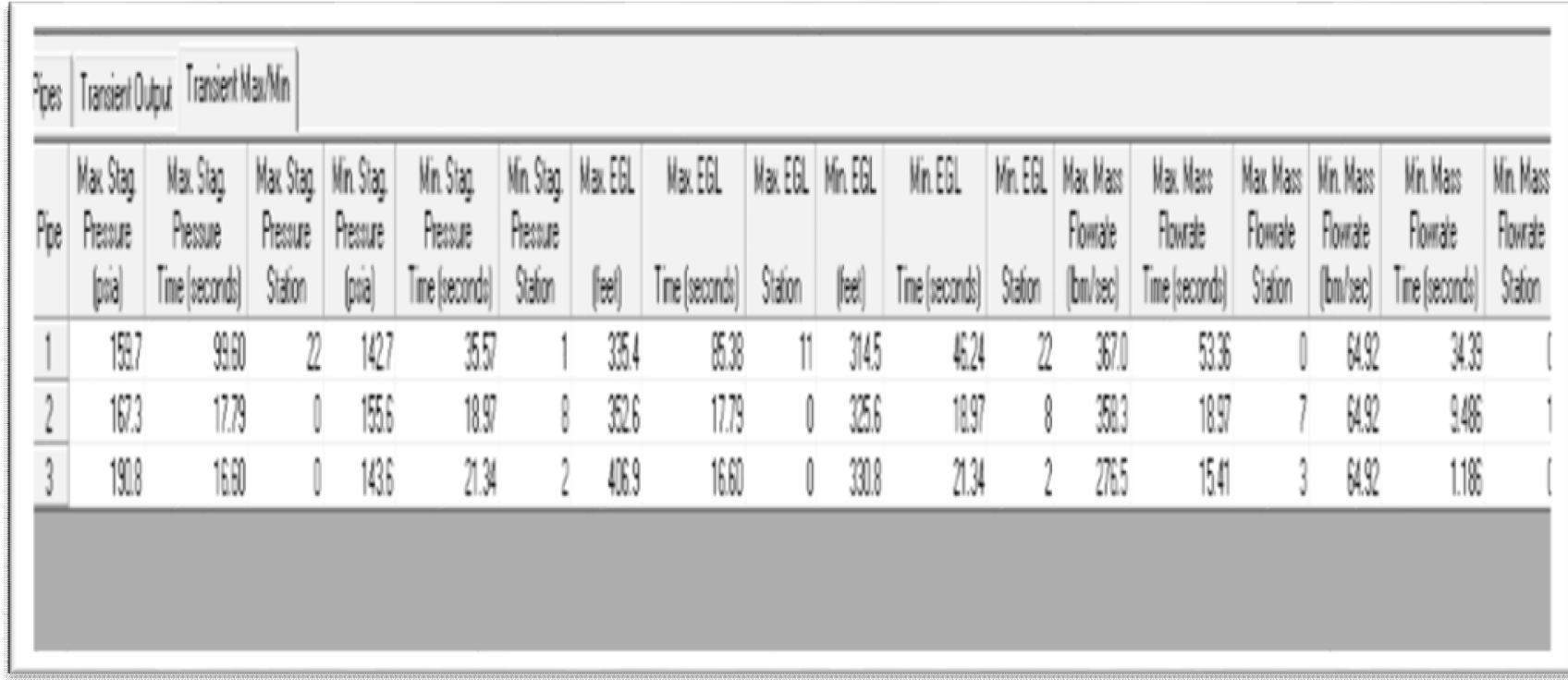

It can be seen from the above two tables that the maximum value of the three-stage pressure pipe has a certain degree of decline under a certain lag control of the time lag, which proves the validity of the time difference control method. By comparing the water hammer analysis in the pipe However, due to the fact that the control of pump station not only considers the safety of pipeline water supply from the aspects of start-up and flow adjustment, Under extreme conditions, considering the safety feasibility of the entire water delivery system, this paper presents a small buffer pool long-distance water delivery system to reduce the buffer pool area to a certain percentage, while ensuring the reduction of land acquisition area, but also for pumping stations The safe operation provides a certain amount of fault tolerance. Next, analyze the time difference control of adjacent pumping stations in the case of small buffer pools. 
The following table 1,2,3,4, respectively, for different proportions (original size buffer pool, covers an area of one-third, covers an area of one-fifth, covers an area of One-tenth) to reduce the buffer pool area, the corresponding adjacent pumping station open time difference simulation.

Table 1 The actual startup status of the original buffer pool

\begin{tabular}{|c|c|c|c|c|}
\hline $\begin{array}{c}\text { Pumping } \\
\text { station }\end{array}$ & $\begin{array}{c}\text { Superior } \\
\text { pump station } \\
\text { traffic } Q_{1} \\
\left(\mathrm{~m}^{3} / \mathrm{s}\right)\end{array}$ & $\begin{array}{c}\text { Buffer pool } \\
\text { footprint }\left(\mathrm{m}^{2}\right)\end{array}$ & $\begin{array}{c}\text { Pipeline surge } \\
\text { propagation } \\
\text { time } T_{F}(\mathrm{~s})\end{array}$ & $\begin{array}{c}\text { Allow to open } \\
\text { pump time } \\
\text { difference }(\mathrm{s})\end{array}$ \\
\hline 1 & 1.95 & 120 & 28.415 & 0 \\
\hline 2 & 1.95 & 70 & 10.716 & 0 \\
\hline 3 & 1.95 & 129.96 & 5.298 & 0 \\
\hline
\end{tabular}

Table 2 Buffer pool footprint reduced to $1 / 3$

\begin{tabular}{|c|c|c|c|c|}
\hline $\begin{array}{c}\text { Pumping } \\
\text { station }\end{array}$ & $\begin{array}{c}\text { Superior } \\
\text { pump station } \\
\text { traffic } Q_{1} \\
\left(\mathrm{~m}^{3} / s\right)\end{array}$ & $\begin{array}{c}\text { Buffer pool } \\
\text { footprint } \\
\left(\mathrm{m}^{2}\right)\end{array}$ & $\begin{array}{c}\text { Pipeline surge } \\
\text { propagation } \\
\text { time } T_{p}(\mathrm{~s})\end{array}$ & $\begin{array}{c}\text { Allow to open } \\
\text { pump time } \\
\text { difference }(\mathrm{s})\end{array}$ \\
\hline 1 & 1.95 & 40 & 28.415 & 53.636 \\
\hline 2 & 1.95 & 23.334 & 10.716 & 37.148 \\
\hline 3 & 1.95 & 43.32 & 5.298 & 82.463 \\
\hline
\end{tabular}

Table 3 Buffer pool footprint reduced to $1 / 5$

\begin{tabular}{|c|c|c|c|c|}
\hline $\begin{array}{c}\text { Pumping } \\
\text { station }\end{array}$ & $\begin{array}{c}\text { Superior pump } \\
\text { station traffic } \\
Q_{1}\left(\mathrm{~m}^{2} / \mathrm{s}\right)\end{array}$ & $\begin{array}{c}\text { Buffer pool } \\
\text { footprint }\left(\mathrm{m}^{2}\right)\end{array}$ & $\begin{array}{c}\text { Pipeline surge } \\
\text { propagation } \\
\text { time } T_{F}(\mathrm{~s})\end{array}$ & $\begin{array}{c}\text { Allow to open } \\
\text { pump time } \\
\text { difference }(\mathrm{s})\end{array}$ \\
\hline 1 & 1.95 & 24 & 28.415 & 20.816 \\
\hline 2 & 1.95 & 14 & 10.716 & 20.053 \\
\hline 3 & 1.95 & 25.992 & 5.298 & 48.019 \\
\hline
\end{tabular}

Table 4 Buffer pool footprint reduced to $1 / 10$

\begin{tabular}{|c|c|c|c|c|}
\hline $\begin{array}{c}\text { Pumping } \\
\text { station }\end{array}$ & $\begin{array}{c}\text { Superior pump } \\
\text { station traffic } \\
Q_{1}\left(\mathrm{~m}^{3} / \mathrm{s}\right)\end{array}$ & $\begin{array}{c}\text { Buffer pool } \\
\text { footprint }\left(\mathrm{m}^{2}\right)\end{array}$ & $\begin{array}{c}\text { Pipeline surge } \\
\text { propagation } \\
\text { time } T_{p}(\mathrm{~s})\end{array}$ & $\begin{array}{c}\text { Allow to open } \\
\text { pump time } \\
\text { difference }(\mathrm{s})\end{array}$ \\
\hline 1 & 1.95 & 12 & 28.415 & 0 \\
\hline 2 & 1.95 & 7 & 10.716 & 3.643 \\
\hline 3 & 1.95 & 12.996 & 5.298 & 32.361 \\
\hline
\end{tabular}

\section{Conclusion}

Based on the current research work of long distance multi-stage pump station water conveyance project, the modeling and analysis of the adjacent two-stage pumping station and its water conveyance pipelines are carried out according to the system control between adjacent pumping stations, According to the principle of water wave propagation, solve the calculation of the time when the water level of the upper water level reaches the next level. According to the principle of water balance, The formula for calculating the allowable opening time difference between two adjacent pumping stations in a long-distance water delivery system for a small buffer pool is deduced. Then, 
the control strategy for the water conveyance is solved. From the formula in Chapter 2, Adjacent stations pumping station propagation time, and then use AFT impulse software to simulate the same time change the adjacent two pumping station operating conditions and the time difference between the two adjacent pumping stations to change the conditions of the corresponding pipe pressure conditions, that The time difference control method is feasible. On this basis, the formula of time difference control method of adjacent pumping stations is used to calculate the maximum allowable difference of power-on time between adjacent pumping stations with different proportions of buffer pool.

\section{References}

[1] Pan.According to the calculation and test of hydraulic transition of Dongshen water supply project [D]. Nanjing: Hohai University, 2005.

[2] HAN Yan-cheng, GAO Xue-ping.A two-step method for automatic control of long-distance gravity flow water conveyance [J] .Journal of Water Science, 2006, 17 (3): 414-418.

[3] Han Yancheng, Gao Xueping. Optimal control mathematical model of long distance water diversion project [J]. Water Resources and Hydropower Engineering, 2005,36 (10): 62-66.

[4] Gao Xueping, Nie Xiaodong, Sun Bowen, et al.Study on the open time difference of adjacent cascade pumping stations in water diversion project [J]. Journal of Hydraulic Engineering, 2016,47 (12): $1502 \sim 1509$

[5] Ben Yue. Simulation Study on Normal Pumping Process of Circulating Water Supply System in a Power Plant [J]. Water Supply Technology, 2010,4 (6): 17-20

[6] Gao Xuezhen, Ben Yue, Yang Yu Si, Shi Qian. Water Hammer Simulation Research on Water Supply of Different Water Supply Systems in a Power Plant. Water supply technology [J], 2011. 5 (4): 21.23 